Tsupykov O. M.

Bogomoletz Institute of Physiology NAS of Ukraine, Kyiv, Ukraine

State Institute of Genetic and Regenerative Medicine NAMS of Ukraine, Kyiv, Ukraine

e-mail: oleg_tsupikov@mail.ru

\title{
NEURAL STEM CELL NICHES IN THE ADULT MAMMALIAN BRAIN
}

\section{ABSTRACT}

Stem cells of the central nervous system have received a great deal of attention in neurobiology in the last decade. It has been shown that neurogenesis occurs in the postnatal period in specialized niches of the adult mammalian brain. The niche is a key regulator of stem cell behavior. Recent data underscore the complexity and heterogeneity of the different components of the niche, and the presence of local signaling microdomain. The review is devoted to recent views on the structural organization of neurogenic niches and regulatory factors involved at different stages of neurogenesis in the postnatal period. Understanding of stem cells behavior in the niches can serve as a basis for determination of these cells function in the adult brain.

KEYWORDS: neural progenitors, neurovascular niche, subventricular zone, subgranular zone.

The dogma that the brain cells of an adult organism do not regenerate prevailed for a long time. This thesis deeply rooted among the scientific community, and therefore in 1962 Joseph Altman's discovery of newly formed cells in adult rodents' brain was almost completely ignored [2]. Later neurogenesis has been described in more adult rodents and songbirds [19, 23]. However, these results were failed to reproduce in adult rhesus monkeys and there was an impression that neurogenesis is missing in the primates' postnatal period [44]. It was believed that new neurons can not be formed in the adult brain, because the brain is not able to regenerate after an injury [60].

Only with the advent of new technologies and after the publication of Samuel Weiss' fundamental works in the early 1990s, neurogenesis in the adult brain has become a generally accepted fact [47]. The use of $3 \mathrm{H}$-thymidine, a radioactive nucleotide, which was used to study proliferation by embedding it into the cells during the S-phase of the cell cycle, was replaced by its analogue bromodeoxyuridine (BrdU), which is detected by specific antibodies. Using BrdU for marking newly formed cells by immunohistochemistry, it was shown that progenitor cells isolated from the adult mice brain are capable to proliferate under conditions in vitro and differentiate into neurons and astrocytes [47]. The presence of neural genesis in the brain of adults was confirmed in 1998, when Fred Gage and Swedish neurobiologist Peter Eriksson first demonstrated the formation of new neurons in the human hippocampus [14]. Today there is no doubt about the existence of permanent neurogenesis in some areas of the brain of adult mammals, which is provided by a pool of neural stem cells (NSCs) $[7,8,32,38,49]$.

Despite the great progress that has been made in understanding of the neural stem cells biology, it is still no clear criteria for identifying the NSCs, which leads to contradictory results about the establishing of the localization of these cells in the postnatal brain, their properties and potential for proliferation and differentiation [10].

Neural stem cells have a large proliferative potential, are capable of self-renewal and generation of multipotent progenitors, which later differ- entiate into the three types of nerve tissue cells - neurons, astrocytes and oligodendrocytes [49].

The definition of "stemness» of NSCs is based on both in vivo and in vitro criteria. A method of neurospheres is most often used to determine NSCs in vitro [18]. Isolated NSCs from the adult brain are cultivated in the presence of growth factors and form neurospheres (diameter 50-150 mm) of proliferating nondifferentiated cells [45]. Later these neurospheres can consistently passage to increase their number, or differentiate in neurons as well as in glial cells [40]. These results show two functional properties of stem cells - self-renewal and multypotency.

Identification of NSCs in terms of in vivo is traditionally based on the analysis of the morphology of these cells and their mitotic activity, as well as on the expression of certain genes and protein synthesis. The most frequently used are such NSCs markers: nestin, Sox2, Musashi 1 and 2, Oct4, Nanog and others; but none of them can be used as the sole criterion for NSCs identification [36].

NSCs in the adult brain are not amorphous nondifferentiated cells, have the properties of differentiated astrocytes on ultrastructural, electrophysiological and molecular levels and express astroglial proteins such as glial fibrillary acidic protein (GFAP), glutamate aspartate transporter (GLAST) and others [3, 13, 41]. During the brain development, radial glia is the primary precursor of neurons and glia [4]. In the postnatal development radial glia transforms into mature astrocytes, but some of them remain properties of stem cells [35]. Note that in mammals to the 11th postnatal day, astrocytes around the brain can generate neurospheres, but then this ability is only in astrocytes, which are in some neurogenic areas of the brain of adult mammals [25]. These specialized neural brain areas, which are a key regulator of stem cell behavior in vivo, maintain their selfrenewal and production of differentiated cells, are called niches [16].

David Scadden determines a niche of stem cells as «a specific anatomical formation that regulates behavior of stem cells that participate in tissue regeneration and supports its functioning» [51]. Niche is a dynamic 

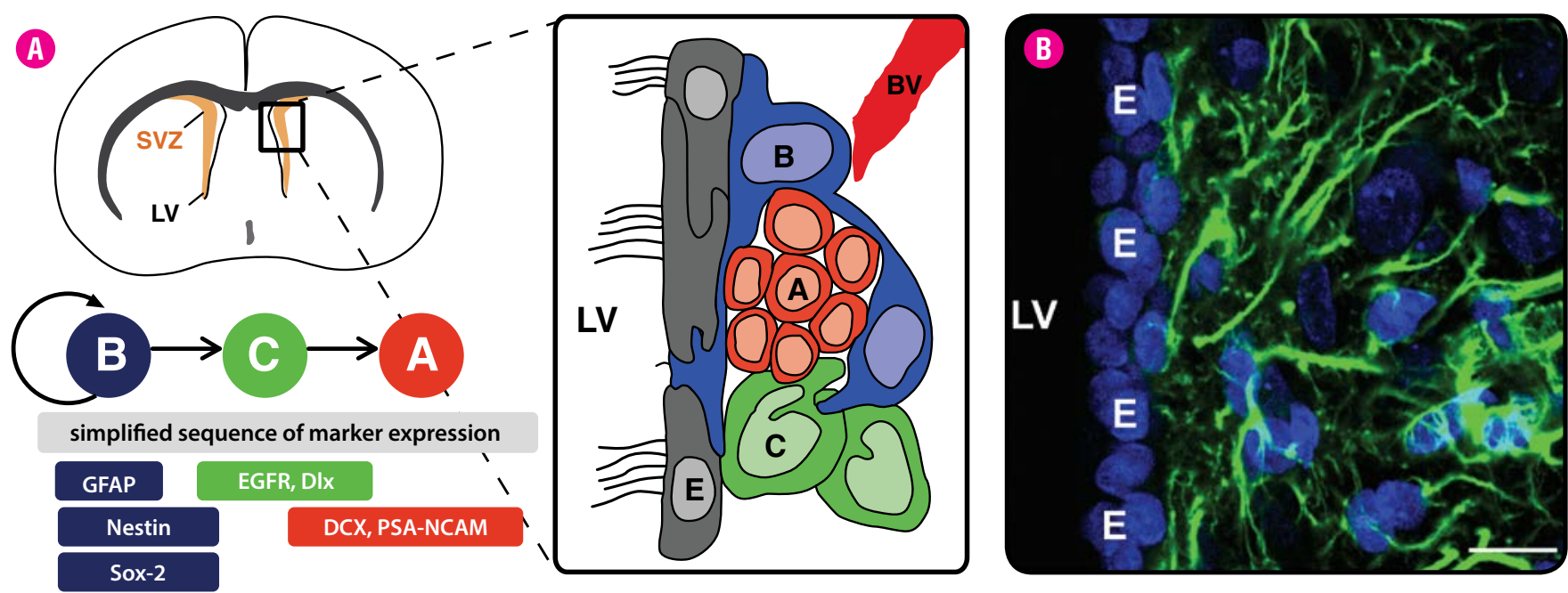

\begin{abstract}
Fig. 1. Structural organization of the adult rodent brain SVZ. A) Schematic representation of subventricular zone (SVZ) adjacent to the lateral ventricles (LV) (modified from [13]). Schematically pictured simplified sequence of expression markers of different SVZ cell types. B - cells type B (SVZ astrocytes), C - type C cells (transit-amplifying cells); A - type A cells (neuroblasts), E - ependymocytes, LV - lateral ventricle, BV - blood vessel. B) Confocal image of rat SVZ. Immunohistochemical staining of astrocytes (GFAP - green). The nuclei of cells stained Hoechst 33342 (blue). LV - lateral ventricle, E ependymocytes. Scale $=10$ microns.
\end{abstract}

system that provides a balanced response of stem cells to the body needs and at the same time protects it from excessive proliferation of stem cells.

Until recently, only two areas of an adult brain were considered as neural stem cell niches. They are subventricular zone (SVZ) of the lateral ventricles and subgranular zone (SGZ) of the dentate gyrus [27]. But in recent years there have apppeared more data that neuro- and gliogenesis in the postnatal brain is really occurs along the entire ventricular system, especially after brain damage or other inducing stimuli $[9,11,26,30,31,48,50]$.

\section{ANATOMY OF SUBVENTRICULAR ZONE}

Subventricular zone extends along the lateral wall of the lateral ventricle and contains a thin layer of cells that retain high proliferative activity. SVZ is the largest germinal zone in the postnatal brain. Location and postnatal proliferative activity of SVZ gives grounds to believe that it is derived from fetal SVZ of lateral ganglionic eminence [58]. Cytoarchitectonics of SVZ is best visualized on front slices of the brain (Fig. 1).

In the subventricular zone of lateral ventricles 4 main types of cells are distinguished: neuroblasts (type A cells), SVZ astrocytes (type B cells), immature progenitor cells (type $C$ cells) and ciliated ependymocytes (type E cells), which stimulate the production of liquor (Fig. 1, A). Slowly proliferating type B cells produce a pool of transitional actively proliferating type $C$ cells (transit amplifying cells), which in its turn generate type A cells (neuroblasts) [12]. In many organs stem cells divide relatively slowly and form a pool of transitional cells that rapidly proliferate, increasing their number before the formation of more mature cells. Such hierarchy of division and differentiation can increase the number of mature cells derived from a single stem cell, thereby minimizing the possibility of mutations during DNA replication in the genome of longlived stem cells [46].

Type B adult NSCs express not only GFAP, GLAST but also express other proteins that are characteristic for mature astrocytes (Fig. 1, B). [12] Despite such glial characteristics of these cells, they are considered to be primary neural stem cells [41]. These cells closely contact with all other types of SVZ cells, including type $C$ cells and neuroblasts (Fig. 1, A) [28]. Moreover, SVZ astrocytes (type B cell) have close contact with blood vessels (Fig. 1, A) and thus create a microenvironment that stimulates neurogenesis [28]. Astrocytic terminal processes (astrocytic endfeet) surrounding blood vessels can be visualized using immunohistochemical staining on Aquaporin-4 (AQP-4) - channels in the membrane of astrocytic endfeet responsible for water permeability. SVZ has small areas on vessels that are not stained with AQP-4, and it is in these areas EGFR-expressing (type $C$ cells) are often in contact with vessels. Such AQP4-negative areas are unique only to SVZ, whereas the cortex and striatum vessels are fully covered with astrocytic endfeet [57]. Blood vessel cells are key elements of stem cell niches in the area of lateral ventricles [27]. An extensive network of blood vessels covers the entire SVZ and closely contacts with NSCs $[27,59]$.

Cells type $B$ and $C$ create a tubular network, along which combined in chains of neuroblasts (type A) migrate through the rostral migratory stream (RMS) to the olfactory bulb, where newly created neurons differentiate into granular and periglomerular neurons [20].

In addition, gliogenesis is also happening in postnatal SVZ; particularly in this area new oligodendrocytes can form as under normal conditions as during remyelinisation [37].

\section{ANATOMY OF SUBGRANULAR ZONE OF DENTATE GYRUS}

Another area of neurogenesis in the adult brain is a subgranular zone of the dentate gyrus. In contrast to the large distances that neuroblasts have to overcome from SVZ to reach their destination - the olfactory bulbs, granular neurons of the dentate gyrus are formed directly in the subgranular area and move on a small distance from SVZ to a layer of granular cells (Fig. 2, A) [24].

The subgranular zone contains SGZ astrocytes (cells type 1), which like SVZ astrocytes are the primary neural stem cells and share morphological and antigenic characteristics with radial glia. These cells usually direct their apical process to the molecular layer and contact with a compulsory component of neurogenic niches - blood vessels (Fig. 2, A, B) [24, 56].

Despite the considerable number of cells type 1 in the subgranular zone, these cells divide slowly. SGZ astrocytes express GFAP, nestin and on ultrastructural level have features peculiar to differentiated astrocytes, but do not express the calcium-binding protein $S 100 \beta$, characteristic of postmitotic population of mature astrocytes $[53,56]$. It has been shown that in addition to neurogenesis, gliogenesis also takes place in the dentate gyrus [53]. Unlike cells type 1, newly postnatal astrocytes of SGZ express not only GFAP, but $S 100 \beta$ [53].

SGZ astrocytes (cells type 1) divide, forming transitional actively proliferating cells (cells type 2). These cells have a small soma and short 

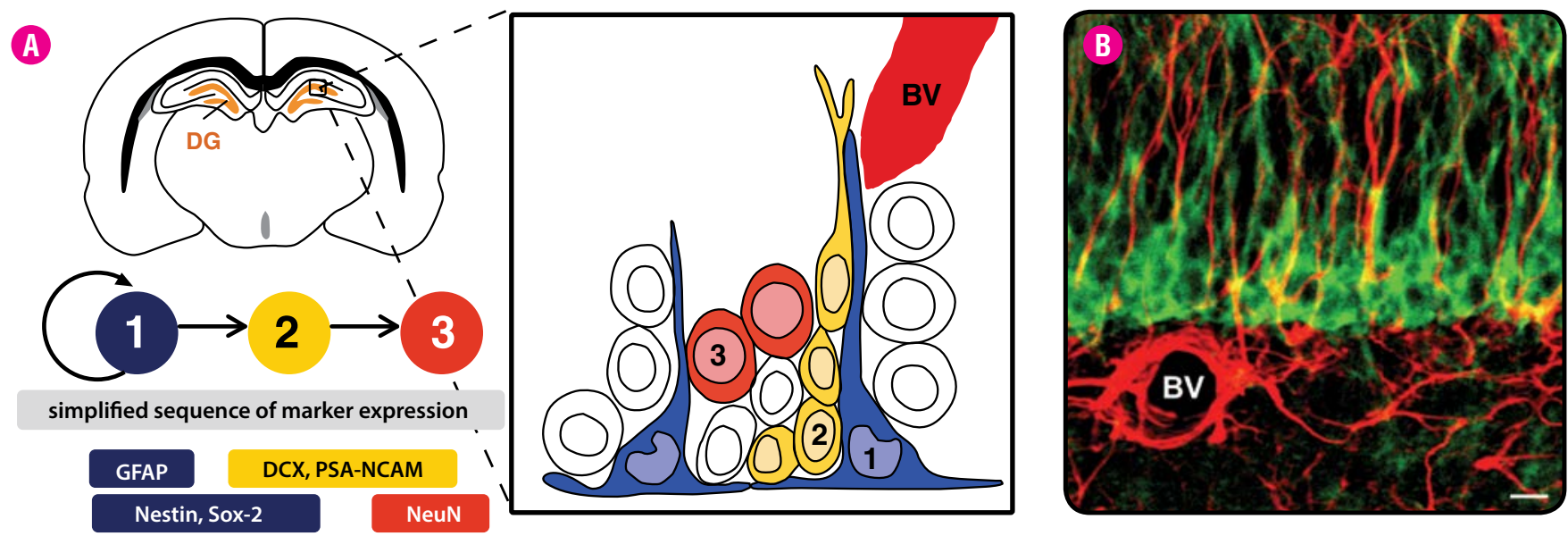

Fig. 2. Structural organization of SGZ of the adult rodent brain. A) Schematic representation of subgranular zone (SGZ) (modified from [13]). Schematically pictured simplified sequence of expression markers of different SGZ cell types. DG - dentate gyrus, 1 - type 1 cells, 2 - type 2 cell, 3 - type 3 cell, BV blood vessel. B) Confocal image of murine SGZ. Immunohistochemical staining of astrocytes (GFAP - red) and progenitor cells (DCX - green). BV - blood vessel. Scale $=10$ microns

processes, they have no apical process as in cells type 1. The cells type 2 express both glial (nestyn) and neuronal (markers DCX, PSA-NCAM) [53]. Cells type 2 are divided into two subtypes, both nestyn positive, one negative (type $2 \mathrm{a}$ ) and the other positive (type $2 \mathrm{~b}$ ) to immature neuronal marker doublecortin (DCX) [5, 24].

Cells type 2 produce slow-proliferating neuroblasts (cells 3 type), which express neuronal markers (DCX, PSA-NCAM, NeuroD, Prox1) and do not express glial markers, indicating the exit from the cell cycle and the beginning of neuronal development [24, 42]. At this stage it is the end for radial cell migration to the granular layer and orientation of cells type 3 processes changes from horizontal to vertical. Leaving the cell cycle is also referred to the intermediate expression of calcium-binding protein calretinin [5]. Mature postmitotic granular neurons are formed from the cells 3 type (Fig. 3A).

So, using the classical concept of stem cells biology in the adult SGZ there are four types of cells with certain properties of progenitor cells: type 1 cells can be considered stem cells; transitional actively proliferating type $2 \mathrm{a}$ and $2 \mathrm{~b}$ progenitor cells (transit-amplifying; and type 3 cells- neuroblasts [24].

Note that postnatal neurogenesis in SGZ is significantly different from neurogenesis in SVZ and inducers of SGZ neurogenesis do not always affect SVZ neurogenesis [6]. Also SVZ progenitor cells are not identical in SGZ cells [52].

Even transplanted in the brain stem cells form ectopic niches of neural stem cells around blood vessels. In our previous studies [21] we have shown that transplanted in rat somato-sensory cortex neural progenitor cells, which overexpressed fibroblast growth factor-2 (FGF-2), formed roundvascular clusters and contacted with endothelial cells through astrocytic end endfeet in areas without pericytes (Fig. 3).

Such NSCs transformation towards FGF-2 overexpression may be a useful strategy to improve integration of transplanted neural progenitors into recipient tissue, thus forming clusters with vascular neurogenic potential for recovery of neural tissue after brain injury [21].

\section{SIGNALLING}

Activation of neural stem cells in the niche, their selfrenewal, proliferation and differentiation is regulated by many signals, such as BMPs and their antagonists Noggin, FGF-2, EGF, Wnt/ $\beta$-catenin, Notch and Shh [13, 17,20 ]. Selfrenewal of NSCs is regulated with Notch signaling [17], while mitogenic EGFR signaling impacts on proliferation and migration of NSCs [55]. Aguirre and others have shown that Notch and EGFR control the number of NSCs and neural progenitor cells (NPCs) [1]. Transcription fac- tor DIx2 is involved in neurogenesis both during embryonic development and in the adult body, and is expressed in type $C$ cells and SVZ neuroblasts [22]. In contrast to neurogenic factors signaling from the family of BMP proteins (bone morphogenetic protein) directs NSCs/NPCs to glial differentiation [29].

An interesting feature of neurogenic niche in both SVZ and SGZ is a close relationship of NSCs with blood vessels; therefore it is called a neurovascular niche [27]. Abundant vascularization of neurogenic niches indicates that soluble factors that distinguish endothelial cells control not only angiogenesis; they can also stimulate neurogenesis and selfrenewal of embryonic and adult NSCs [27, 34, 39, 57]. For example, intraventricular administration of vascular endothelial growth factor (VEGF), which is a major angiogenic factor secreted by endothelial cells, also increases NSCs proliferation in SVZ and SGZ [54]. Synergetic interaction between VEGF and FGF-2 signaling may underlie the coordination of angiogenesis and neurogenesis in SVZ and SGZ [39]. Besides VEGF, endothelial cells produce other signals that affect NSCs: Notch, FGF2, IGF-I, PEDF, BDNF and others. [43].

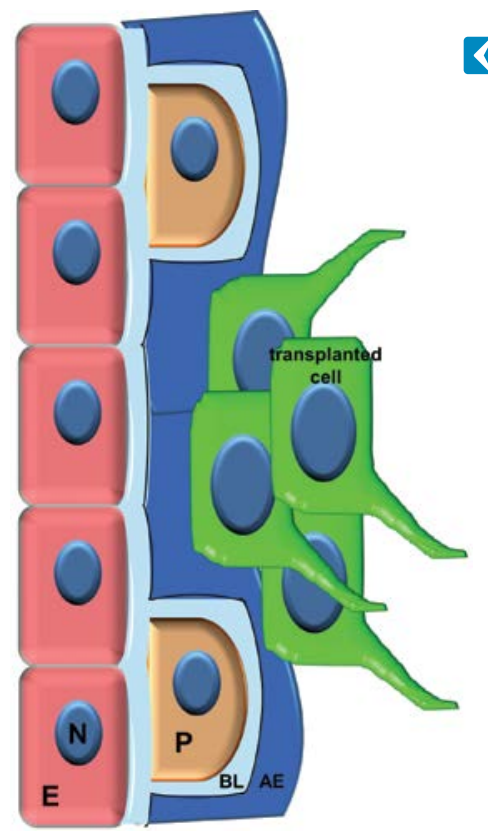

Fig 3. A schematic drawing that shows the structural organization of ectopic neurogenic niches of stem cells. $A E$ - astrocytic endfeet, $\mathrm{BL}$ - basal lamina, E - endothelial cells, $P$ - pericyte, TC - transplanted cell, $\mathrm{N}-$ the cell nucleus. 
A neurovascular niche includes not only NSCs, but also a variety of differentiated cells, such as glial cells, neurons and extracellular matrix [33]. An integral part of a neurovascular niche is a basal lamina (BL) and extracellular matrix (EM) components that reinforce cells and provide them with a variety of factors [33]. Regulation of neurogenic niche functions takes place also due to EM proteins involved in both proliferation and migration of NSCs, namely collagen-1, tenascin C, heparan sulfate proteoglycan, chondroitin sulfate proteoglycan, laminin a2 and
B1 integrins [15]. For example, proteoglycan heparan sulfat bind growth factors (EGFs, FGFs, IGF-II, PDGF-AA and VEGF), morphogens and mitogens (BMP-2, BMP-4, Shh and Wnts), chemokines and cytokines [15, $29,34]$. It was shown that in neurovascular niches basal lamina forms labyrinth-like branched structures - fractons $[13,34]$. In SVZ fractons cover all types of cells including NSCs and promote cell proliferation by binding to the extracellular environment of neurogenic factors such as FGF-2 [34].

\section{CONCLUSION}

Specialized brain environment or niche regulates selfrenewal, activation and differentiation of neural stem cells and enhances neurogenesis after brain damage or influenced by other inducing factors. Common components of neurovascular niche of stem cells are endothelial cells, signaling from the somatic cells, basement membrane for attaching the cells and extracellular matrix, which modulates the adhesiveness and activity of signaling molecules. Analysis of the behavior of stem cells in neurovascular niches can serve as a basis for understanding of these cells functions in the adult mammalian brain.

\section{REFERENCES}

1. Aguirre A, Rubio ME, Gallo V. Notch and EGFR pathway interaction regulates neural stem cell number and self-renewal. Nature. 2010; 467(7313):323-327.

2. Altman J. Are new neurons formed in the brains of adult mammals? Science. 1962; 135(3509):1127-1128.

3. Alvarez-Buylla A, Garcia-Verdugo JM, Tramontin AD. A unified hypothesis on the lineage of neural stem cells. Nat. Rev. Neurosci. 2001; 2:287-293.

4. Anthony TE, Klein C, Fishell G, et al. Radial glia serve as neuronal progenitors in all regions of the central nervous system. Neuron. 2004; 41:881-890.

5. Brandt MD, Jessberger $S$, Steiner $B$, et al. Transient calretinin expression defines early postmitotic step of neuronal differentiation in adult hippocampal neurogenesis of mice. Mol Cell Neurosci. 2003; 24:603-613.

6. Brown J, Cooper-Kuhn CM, Kempermann G, et al. Enriched environment and physical activity stimulate hippocampal but not olfactory bulb neurogenesis. Eur. J. Neurosci. 2003; 17(10):2042-2046.

7. Capilla-Gonzalez V, Lavell E, Quiñones-Hinojosa A, et al. Regulation of subventricular zone-derived cells migration in the adult brain. Adv. Exp. Med. Biol. 2015; 853:1-21.

8. Costa V, Lugert S, Jagasia R. Role of adult hippocampal neurogenesis in cognition in physiology and disease: pharmacological targets and biomarkers. Handb. Exp. Pharmacol. 2015; 228:99-155.

9. Cunningham LA, Candelario K, Li L. Roles for HIF-1a in neural stem cell function and the regenerative response to stroke. Behav. Brain Res. 2012; 227:410-417.

10. Decimo I, Bifari F, Krampera M, et al. Neural stem cell niches in health and diseases. Curr. Pharm. Des. 2012; 18(13):1755-1783.

11. Decimo I, Bifari F, Rodriguez FJ, et al. Nestin- and doublecortin-positive cells reside in adult spinal cord meninges and participate in injury-induced parenchymal reaction. Stem Cells. 2011;29:2062-2076.

12. Doetsch F. A niche for adult neural stem cells. Curr. Opin. Genet. Dev. 2003; 13:543-550.

13. Doetsch F. The glial identity of neural stem cells. Nat.Neurosci. 2003; 6:1127-1134.

14. Eriksson PS, Perfilieva E, Björk-Eriksson T, et al. Neurogenesis in the adult human hippocampus. Nat. Med. 1998; 4(11):1313-1317.

15. Faissner A, Reinhard J. The extracellular matrix compartment of neural stem and glial progenitor cells. Glia. 2015; 63(8):1330-1349.

16. Fuchs E, Tumbar T, Guasch G. Socializing with the neighbors: stem cells and their niche. Cell. 2004; 116:769-778.

17. Giachino C, Taylor V. Notching up neural stem cell homogeneity in homeostasis and disease. Front. Neurosci. 2014; 8:32.

18. Gil-Perotín S, Duran-Moreno M, Cebrián-Silla A, et al. Adult neural stem cells from the subventricular zone: a review of the neurosphere assay. Anat. Rec. (Hoboken). 2013; 296(9):1435-1452.

19. Goldman SA, Nottebohm F. Neuronal production, migration, and differentiation in vocal control nucleus of the adult female canary brain. Proc. Natl. Acad. Sci. USA. 1983; 80:2390-2394.

20. Hirota $Y$, Sawada M, Huang SH, et al. Roles of Wnt signaling in the neurogenic niche of the adult mouse ventricular-subventricular zone. Neurochem. Res. 2015; Nov 16. [Epub ahead of print].

21. Jenny B, Kanemitsu $M$, Tsupykov 0 , et al. Fibroblast growth factor-2 overexpression in transplanted neural progenitors promotes perivascular cluster formation with a neurogenic potential. Stem Cells. 2009; 27(6):1309-1317.

22. Jones KS, Connor B. Intrinsic regulation of adult subventricular zone neural progenitor cells and the effect of brain injury. Am. J. Stem Cells. 2011; 1(1):48-58.

23. Kaplan MS, Hinds JW. Neurogenesis in the adult rat: electron microscopic analysis of light radioautographs. Science. 1977; 197:1092-1094.

24. Kempermann G, Song H, Gage FH. Neurogenesis in the adult hippocampus. Cold Spring Harb. Perspect. Med. 2015; 5(7):a018812.

25. Laywell ED, Rakic P, Kukekov VG, et al. Identification of a multipotent astrocytic stem cell in the immature and adult mouse brain. Proc. Natl Acad. Sci. USA. 2000; 97:13883-13888

26. Lee SW, Clemenson GD, Gage FH. New neurons in an aged brai. Behav. Brain Res. 2012; 227:497-507.

27. Licht $T$, Keshet $E$. The vascular niche in adult neurogenesis. Mech. Dev. 2015; 138P1:56-62.

28. Lim DA, Alvarez-Buylla A. Adult neural stem cells stake their ground. Trends Neurosci. 2014; 37(10):563-571. 
29. Lim DA, Tramontin AD, Trevejo JM, et al. Noggin antagonizes BMP signaling to create a niche for adult neurogenesis. Neuron. 2000; 28(3):713-726.

30. Lin R, lacovitti L. Classic and novel stem cell niches in brain homeostasis and repair. Brain Res. 2015; S0006-8993(15)00325-X.

31. Lin R, Cai J, Nathan $C$, et al. Neurogenesis is enhanced by stroke in multiple new stem cell niches along the ventricular system at sites of high BBB permeability. Neurobiol. Dis. 2015; 74:229-239.

32. Mariano ED, Teixeira MJ, Marie SK, et al. Adult stem cells in neural repair: Current options, limitations and perspectives. World J. Stem Cells. 2015; 7(2):477-482.

33. Massirer KB, Carromeu C, Griesi-Oliveira K, et al. Maintenance and differentiation of neural stem cells. Wiley Interdiscip Rev. Syst. Biol. Med. $2011 ; 3(1): 107-114$.

34. Mercier F, Kitasako JT, Hatton GI. Anatomy of the brain neurogenic zones revisited: fractones and the fibroblast/macrophage network. J. Comp. Neurol. 2002; 451:170-188.

35. Merkle FT, Tramontin AD, Garcia-Verdugo JM, et al. Radial glia give rise to adult neural stem cells in the subventricular zone. Proc. Natl. Acad. Sci. USA. 2004; 101:17528-17532.

36. Montalbán-Loro R, Domingo-Muelas A, Bizy A, et al. Epigenetic regulation of stemness maintenance in the neurogenic niches. World J. Stem Cells. 2015; 7(4):700-710.

37. Nait-Oumesmar B, Decker L, Lachapelle F, et al. Progenitor cells of the adult mouse subventricular zone proliferate, migrate and differentiate into oligodendrocytes after demyelination. Eur. J. Neurosci. 1999; 11:4357-4366.

38. Nam H, Lee KH, Nam DH, et al. Adult human neural stem cell therapeutics: Current developmental status and prospect. World J. Stem Cells. $2015 ; 7(1): 126-136$.

39. Ottone $C$, Krusche B, Whitby A, et al. Direct cell-cell contact with the vascular niche maintains quiescent neural stem cells. Nature Cell Biol. 2014; 16:1045-1056.

40. Park K, Nam Y, Choi Y. An agarose gel-based neurosphere culture system leads to enrichment of neuronal lineage cells in vitro. In Vitro Cell Dev. Biol. Anim. 2015; 51(5):455-462.

41. Platel JC, Bordey $A$. The multifaceted subventricular zone astrocyte: From a metabolic and pro-neurogenic role to acting as a neural stem cell. Neuroscience. 2015. pii: S0306-4522(15)00981-1.

42. Plumpe T, Ehninger D, Steiner B, et al. Variability of doublecortin-associated dendrite maturation in adult hippocampal neurogenesis is independent of the regulation of precursor cell proliferation. BMC Neurosci. 2006; 7:77.

43. Putnam AJ. The instructive role of the vasculature in stem cell niches. Biomat. Sci. 2014; 2:1562-1573.

44. Rakic $P$. Limits of neurogenesis in primates. Science. 1985; 227(4690):1054-1056.

45. Ramasamy S, Narayanan G, Sankaran S, et al. Neural stem cell survival factors. Arch. Biochem. Biophys. 2013; 534(1-2):71-87.

46. Reya T, Morrison SJ, Clarke MF, et al. Stem cells, cancer, and cancer stem cells. Nature. 2001; 105-111.

47. Reynolds $B A$, Weiss $S$. Generation of neurons and astrocytes from isolated cells of the adult mammalian central nervous system. Science. 1992; 255:1707-1710.

48. Robins SC, Stewart I, MCNay DE, et al. a-Tanycytes of the adult hypothalamic third ventricle include distinct populations of FGF-responsive neural progenitors. Nat. Commun. 2013; 4:2049.

49. Rueger MA, Schroeter M. In vivo imaging of endogenous neural stem cells in the adult brain. World J. Stem Cells. 2015; 7(1):75-83.

50. Sanin V, Heeß C, Kretzschmar HA, Schuller U. Recruitment of neural precursor cells from circumventricular organs of patients with cerebral ischaemia. Neuropathol. Appl. Neurobiol. 2013; 39:510-518.

51. Scadden D.T. The stem-cell niche as an entity of action. Nature. 2006; 441(7097):1075-1079.

52. Seaberg RM, van der Kooy DJ. Adult rodent neurogenic regions: the ventricular subependyma contains neural stem cells, but the dentate gyrus contains restricted progenitors. Neurosci. 2002; 22(5):1784-1793.

53. Steiner B, Kronenberg G, Jessberger S, et al. Differential regulation of gliogenesis in the context of adult hippocampal neurogenesis in mice. Glia. 2004; 46:41-52.

54. Sun YJ, Jin K, Childs JT, et al. Vascular endothelial growth factor-B (VEGFB) stimulates neurogenesis: Evidence from knockout mice and growth factor administration. Developmental Biology. 2006; 289(2):329-335.

55. Sütterlin P, Williams EJ, Chambers D, et al. The molecular basis of the cooperation between EGF, FGF and eCB receptors in the regulation of neural stem cell function. Mol. Cell. Neurosci. 2013; 52:20-30.

56. Tao Y, Ma L, Liao Z, et al. Astroglial $\beta$-arrestin1-mediated nuclear signaling regulates the expansion of neural precursor cells in adult hippocampus. Sci. Rep. 2015; 5:15506.

57. Tavazoie M, Van der Veken L, Silva-Vargas V, et al. A specialized vascular niche for adult neural stem cells. Cell Stem Cell. 2008; 3(3):279-288.

58. Tramontin AD, García-Verdugo JM, Lim DA, et al. Postnatal development of radial glia and the ventricular zone (VZ): a continuum of the neural stem cell compartment. Cereb. Cortex. 2003; 13(6):580-587.

59. Urbán N, Guillemot F. Neurogenesis in the embryonic and adult brain: same regulators, different roles. Front. Cell. Neurosci. 2014; 8:396.

60. Whitman MC, Greer CA. Adult neurogenesis and the olfactory system. Prog. Neurobiol. 2009; 89(2):162-175.

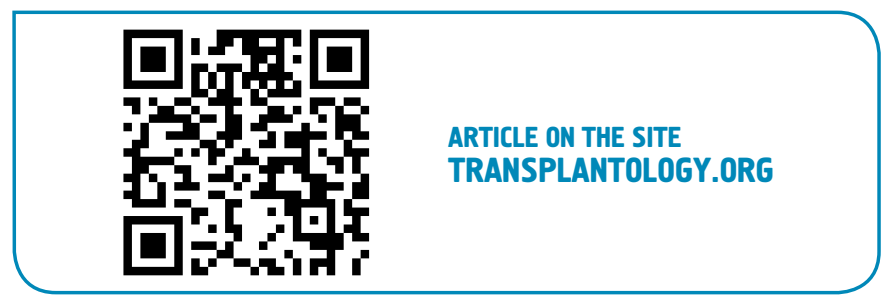

The author indicates no potential conflicts of interest.

Received: September 03, 2015

Accepted: November 16, 2015 